\title{
Set-size effects in primary memory: An age-related capacity limitation?
}

\author{
GRAEME S. HALFORD, MURRAY T. MAYBERY, and JOHN D. BAIN \\ University of Queensland, St. Lucia, Queensland, Australia
}

\begin{abstract}
Set size was varied as a factor in the primary memory paradigm developed by Wickens and colleagues (Wickens, Moody, \& Dow, 1981; Wickens, Moody, \& Vidulich, 1985). In Experiment 1, using adults and teenagers, no proactive interference (PI) was observed at set size 4, consistent with previous research, but PI was observed at set size 10. In Experiment 2, with adults, PI was observed at set sizes 6,8 , and 10 , but not at set size 4 . These results are consistent with the hypothesis that sets of more than 4 items overload primary memory, so that some items have to be retrieved from secondary memory. In Experiment 3, 8- to 9-year-olds were used with set sizes of 2 and 4. Proactive interference was observed with the larger, but not with the smaller, set. This suggests that primary memory was overloaded by 4 items at this age, indicating that its capacity increases with age.
\end{abstract}

Wickens, Moody, and Dow (1981) and Wickens, Moody, and Vidulich (1985) made considerable progress toward operationalizing the distinction, originally made by William James (1890), between primary and secondary memory. As defined by James, primary memory (PM) consists of information that has not been lost to consciousness, whereas secondary memory (SM) consists of information of which we are not currently thinking. Wickens et al. (1981) operationalized PM and SM in the Sternberg (1975) memory scanning task. In the PM condition, a memory set is presented and followed almost immediately by the probe item, without intervening activity. In the SM condition, a distracting activity (counting backward, or a Stroop task) intervenes between memory set presentation and probe presentation, thereby removing the memory set items from consciousness.

Wickens et al. (1981; Wickens et al., 1985) showed that PM and SM are distinguished by three factors in this situation: (1) information in SM has to be retrieved, whereas information in PM does not, and retrieval produces an increment in response time (RT) to the probe; (2) negative probes produce longer RTs than positive probes for SM but not for PM, probably because SM requires retrieval, and negative probes, which do not belong to the memory set, are less effective retrieval cues; and (3) proactive interference effects occur in SM but not in PM, because proactive interference is associated with retrieval.

Wickens et al. (1981) used set sizes of 2 and 4 items under PM and SM conditions. Retrieval latencies were longer in the SM condition by an amount that was indepen-

This work was supported by a grant from the Australian Research Grants Scheme. We would like to thank Alan Baddeley, Delos Wickens, and three anonymous reviewers for helpful comments on an earlier draft of this paper. We also would like to thank Brett Garrigan, Susan Robinson, and Robert Lake for their careful assistance. Address correspondence to Graeme Halford, Department of Psychology, The University of Queensland, St. Lucia 4067, Queensland, Australia. dent of set size, causing Wickens et al. (1981) to conclude that a pointer to the set, not the set itself, is retrieved; that is, it takes no longer to retrieve a larger than a smaller set because it is the pointer to the set, not the individual items, that are retrieved. Wickens et al. (1985) found support for these same conclusions using set sizes of 1,2, and 4 items and using an additional manipulation, extracategory probes, that reduced the scanning rate but left retrieval time unaffected.

Proactive interference (PI) was manipulated by using items from the same semantic category on three successive trials, followed by a change to a new semantic category. The first trial in a new category is low PI, whereas the third trial in a category is high PI. PI effects were found only in the SM condition.

Wickens et al.'s (1981; Wickens et al., 1985) conclusion that there is no PI in the absence of distracting activity between memory set and probe presentation has been validated only for sets of up to 4 items. This conclusion might not hold for larger memory sets. Perhaps PM has a limited capacity, so that with large sets it may not be possible to maintain all items in an active state. In these circumstances, some or all of the items could not be retained in PM, and therefore would have to be retrieved from SM in order to respond to the probe. According to the argument of Wickens et al. (1981), this retrieval would be susceptible to PI. According to our extension of their argument, the larger the set size, the more likely it is that items would be retrieved from SM, and the more likely it is that PI would be observed.

In Experiment 1, Wickens et al.'s (1981; Wickens et al., 1985) PM paradigm was used with set sizes of 4 and 10 items. If a 10 -item memory set exceeds the capacity of PM, some items will have to be held in SM and will be subject to PI effects, but PI should not be observed for sets of 4 items. This argument predicts a set size (4/10) $\times$ PI (high/low) interaction. 


\section{EXPERIMENT 1}

\section{Method}

Participants. Forty-eight volunteer teenagers (mean age $=$ 14.7 years, $S D=1.46$ ), who answered a newspaper advertisement, were paid $\$ 10$ for their participation. Forty-eight first-year psychology undergraduates also participated, to fulfill a course requirement.

Apparatus. The apparatus consisted of a BBC model B microcomputer, a 43-cm Amust color monitor, and a button box with buttons labeled "yes" on the right and "no" on the left.

Materials. The materials for test trials consisted of $\mathbf{4 0}$ taxonomic categories of 27 words each, compiled primarily from the Battig and Montague (1969) norms. Examples of the categories were types of trees, kitchen utensils, sports, diseases, boats, and spices. Words were chosen for high familiarity, maximum intracategory similarity, and minimum intercategory similarity. High-familiarity words in the tree category included pine, chestnut, and palm, and lowfamiliarity items included balsam, cottonwood, and sequoia. The shortest words consistent with the other constraints were used. There were also 120 uncategorized words used in practice problems.

Design and Procedure. The three factors, which were all within subjects, were set size ( 4 items vs. 10 items), proactive interference (high vs. low), and probe type (positive vs. negative).

A single trial consisted of the simultaneous presentation of words in the memory set for a duration equal to $1 \mathrm{sec}$ plus $1.2 \mathrm{sec}$ per word. The words were displayed in a vertical list in the center of the screen. After the screen cleared, a row of asterisks appeared across the center of the screen for $1 \mathrm{sec}$, after which the probe word appeared in the place of the asterisks. Then the participant pressed the "yes" or "no" button, indicating that the probe word was or was not in the memory set. There was a 5-sec intertrial interval.

The 136 trials, 16 practice and 120 test trials, were presented in a single session, but with breaks after 34 trials ( 16 practice and 18 test trials), 67 trials, and 103 trials. There were 3 consecutive test trials using words in one taxonomic category, then 3 consecutive trials using another category, and so on for the 40 categories. The first trial in a given category was low PI, and the third trial in the category was high PI. The 3 trials within a category used one of the following sequences of set sizes: $4-10-4,4-10-10,10-4-4$, or 10-4-10. The purpose was to equate number of items within the category preceding the third trial, and to ensure that each set size would occur equally often in the low- and high-PI conditions. Each sequence was used 10 times. The order of categories and the selection of sets from categories were randomized for each participant.

The probes were selected so that half were positive and half were negative within each combination of high/low PI with each set size, so there were no more than four consecutive positive or negative trials. Probe words were counterbalanced across participants so that a word was used once in each combination of high/low PI, set size, and positive/negative probes. The positive probes sampled each serial position once in set size 10 , and two or three times in set size 4.

\section{Results and Discussion}

Latency. The latency measure was each participant's median latency calculated over the 10 trials in a cell, but restricted to correct trials. These scores were analyzed in a $2 \times 2 \times 2 \times 2($ age $\times$ set size $\times P I \times$ probe type) analysis of variance (ANOVA). This yielded a main effect of set size $[F(1,94)=136.62, M S e=22,548, p<.001]$ and an interaction of set size with PI $[F(1,94)=4.62$, $M S \mathrm{e}=11,412, p<.05]$. These means are shown in Figure 1A. The lack of a PI effect at set size 4 is consistent with Wickens et al. (1981; Wickens et al., 1985), and
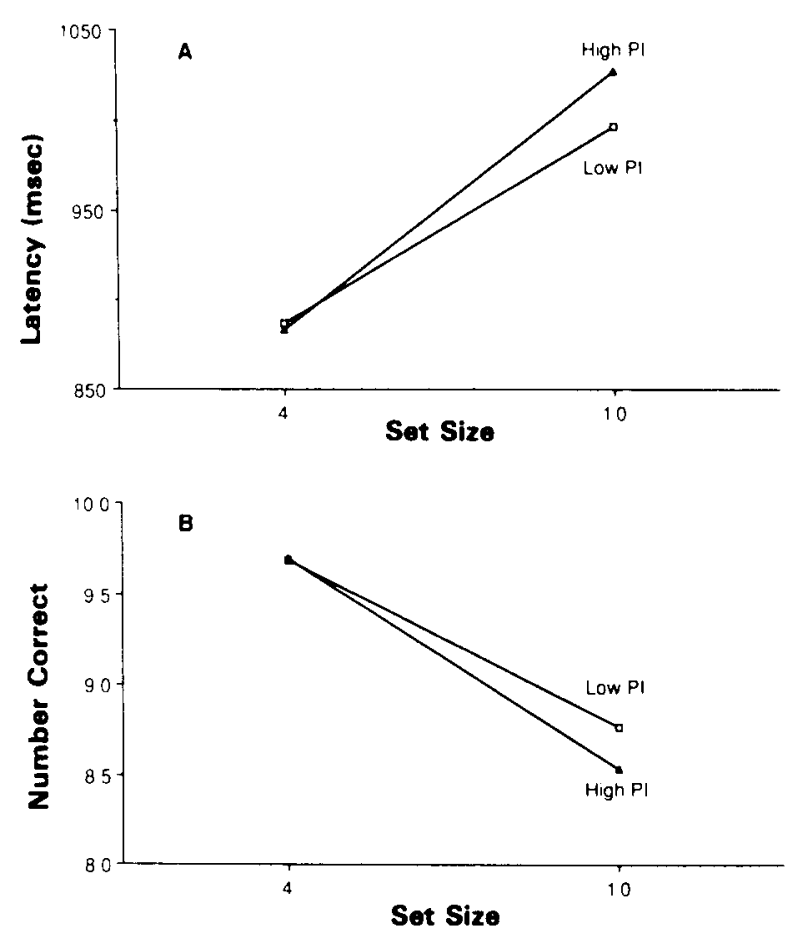

Figure 1. Interaction of set size and proactive interference (PI) for latency (panel A) and number correct (panel B) in Experiment 1.

the finding of a PI effect with large set sizes supports the hypothesis in the introduction; that is, it appears that the 10 -item set exceeds the capacity of PM, with the result that some items are retrieved from SM and are subject to PI. There was also a main effect of age $[F(1,94)=$ $17.84, M S e=475,407, p<.001]$, indicating faster responding by older children, but this was modified by an age $\times$ PI $\times$ probe type interaction $[F(1,94)=5.90$, $M S e=17,328, p<.05]$. Longer latencies were found for negative probes, except for the younger group in the low-PI condition. There is no obvious reason for this pattern of data. The absence of an age $\times$ PI $\times$ set size interaction means there is no evidence in these data that PM capacity varies with age over the (somewhat narrow) range tested.

Accuracy. An ANOVA of the same design as that conducted on latencies was performed on the number of correct responses out of 10 . This yielded a main effect of set size $[F(1,94)=289.80, M S e=.719, p<.001]$ and an interaction of set size and PI $[F(1,94)=4.09, M S \mathrm{e}$ $=.670, p<.05]$. The means are shown in Figure 1B. The interaction supports the hypothesis that a PI effect, although not present at the small set sizes, would be observed with a large set size. Note that the PI effects in accuracy and latency cannot be attributed to a speed-accuracy trade-off: the lowest accuracy (high PI, set size 10) was accompanied by the slowest latency.

There was also a main effect of positive/negative probe type $[F(1,94)=99.21, M S \mathrm{e}=1.378, p<.001]$ and an interaction of this factor with set size $[F(1,94)=78.50$, $M S \mathrm{e}=.847, p<.001]$. Mean proportions correct were 
as follows: for set size 4 , positive probes .95 , negative probes .98; for set size 10 , positive probes .80 , negative probes .95 . The tendency for negative trials to yield more correct responses could reflect a bias toward responding "no" when uncertain. Uncertainty is greater for large set sizes; hence the increased positive/negative difference.

The interaction of set size and PI might not reflect overloading of primary memory with consequent retrieval of items from secondary memory, but instead longer mean retention intervals with larger set sizes. One implication of this hypothesis would be that items in earlier serial positions should be less well recognized because they would have been scanned first and would have been stored for longer. To evaluate this hypothesis, a trends analysis was conducted for set size 10 . Significant linear trends were obtained for latencies $[F(1,855)=4.28, M S e=814,388$, $p<.05]$ and for proportion correct $[F(1,855)=19.56$, $M S \mathrm{e}=.1539, p<.01]$. For latencies, the best-fitting linear trend was RT $=1,172+14.8 S$, where $S$ is serial position. For accuracy, the best-fitting linear trend was $P c=.89-.017 S$, where $P c$ is proportion correct. In both cases, the trend is opposed to the hypothesis; that is, performance is poorer for later serial positions. (We also checked the interaction between serial position and PI, but $F<1$.)

Experiment 1 confirmed the prediction made in the introduction that PI effects do occur with set size 10 but, consistent with Wickens et al. (1981; Wickens et al., 1985), not with set size 4 . This suggests that 10 items may exceed the capacity of PM, requiring some items to be retrieved from SM. The implication is that the capacity of PM for adults and teenage children is somewhere between 4 and 10 items. In Experiment 2 we used set sizes of $4,6,8$, and 10 items to determine more precisely the set size at which PI effects are first observed. We first compared set size 4 with larger sets, then did the same for set size 6 and 8 .

The PI effects were of similar magnitude to those observed by Wickens et al. (1981; Wickens et al., 1985), but it would be desirable to increase the size of the effect so as to gain greater statistical power. To this end, two changes were made for Experiment 2. The first was to use rhyming categories. We reasoned that PI occurs because of difficulty in discriminating parts of the current memory set that may be stored in SM from sets in the same category used on previous trials (Gardiner, Craik, \& Birtwhistle, 1972; Watkins \& Watkins, 1975), and that intracategory similarity could be maximized and extracategory similarity minimized by using rhyming categories. An additional reason for using rhyming categories was that we intended to use children in Experiment 3 with the same procedure, and our previous work had shown that children are less sensitive to semantic category shifts than are adults. Second, memory sets were selected so that the third set within a category shared some items with previous sets from the same category. We anticipated that this would make the current set even less discriminable from sets used on previous trials, thereby magnifying the PI effect in SM. On the other hand, when the whole of the current memory set can be kept active in PM, as occurs with small set sizes, it should retain its discriminability from previous sets. Thus the two alterations should increase PI for items in SM, but not for items in PM, thereby increasing the power of the set size $X$ PI interaction, and should permit smaller, more economical experimental designs to be used.

\section{EXPERIMENT 2}

\section{Method}

Participants. The participants were 64 first-year psychology students, all native English speakers, who fulfilled a course requirement by participating. An additional 5 participants were eliminated because they did not maintain the .80 accuracy criterion, and 1 who was eliminated because he said that on set size 10 he "knew what the words were" and did not read the words presented on the screen.

Apparatus. The apparatus were the same as in Experiment 1.

Materials. The materials consisted of 32 rhyming categories, each of 21 words, based on lists compiled by Walling, McEvoy, Oth, and Nelson (personal communication, 1984). The words were all of two syllables or less, and preference in selection was given to words of high frequency in Australian usage.

Design and Procedure. The factors were set size $(4,6,8$, or 10 items), proactive interference (high/low), probe type (positive/negative), and, nested within the high-PI condition, old/new probe. Old high-PI probes had been used in an earlier set in the same category, whereas new probes had not.

The procedure for a single trial was the same as in Experiment 1 except that the lists were displayed for $1 \mathrm{sec}$ plus $1.5 \mathrm{sec}$ per word. Also, rhyming categories were used instead of semantic categories. Testing took place in three sessions, each of two blocks. Each session lasted approximately $\mathbf{4 0} \mathrm{min}$, there was a break of approximately 5 min between blocks, and sessions were separated by at least $1 \mathrm{~h}$. The first block comprised 33 practice trials, and blocks two through six each comprised 3 practice and 48 test trials. In addition, a span test was administered at the end of the third session.

The first two trials within a category used one of the following sequences of set sizes: $4-10,10-4,6-8$, or $8-6$. The third trial used set size $4,6,8$, or 10 . The 16 resulting combinations were used five times for each participant within the test trials. The purpose was to equate the number of items within the category preceding the third trial, and to ensure that each set size occurred equally often in the low- and high-PI conditions.

Words for the second trial within a category were selected so that approximately $25 \%$ had been used in the first trial, and words for the third trial within a category were selected so that approximately $50 \%$ had been used in the first two trials. Half of the probes for the third trial within a category were "old" in that they had been used in the first or second trial (but not as the probe word), and half were "new" in that they had not been used. All categories were used once in random order, then reused in a new random order, with the restriction that at least one block of trials intervened between repetitions of a category. The positive probes sampled each serial position once in set size 10, once or twice in set sizes 8 and 6 , and two or three times in set size 4 .

Instructions mentioned that words within a list would rhyme, accuracy was emphasized, and participants were encouraged to use the same learning strategy across all set sizes. Extensive feedback was given on the first three practice trials to encourage compliance with these instructions, and to direct attention to the distinction between old and new probes and between positive and negative probes. It was made clear that membership in the current set was the decision to be made; that is, "old" probes from previous trials, but 
those not in the current memory set, were still to be responded to as negative. In other respects, the procedure was the same as in Experiment 1.

The span test. After the probe recognition blocks, a span test was administered to provide a preliminary indication as to whether there is an association between PM capacity and span for equivalent materials. The procedure for a single span trial was the same as in the main body of Experiment 2, except that following the display of the asterisks participants attempted immediate, ordered recall of the word list. The next trial began $4 \mathrm{sec}$ after responding and recording were completed. Words within a trial were from the same hyming category, and those from different trials were from different categories.

The up-down method of span determination was used. A total of 20 trials were given, 5 practice followed by 15 test. Set size 6 was presented in Trial 1. On subsequent trials, set size was determined by performance; set size increased by 1 following a correct response and decreased by 1 following an incorrect response. The span score was the mean length of all trials presented after the practice trials minus .5. (This is because the mean is the average of the strings correct and the strings failed, so it would be .5 item longer than true span.)

\section{Results and Discussion}

Latency. A $4 \times 2 \times 2$ (set size $\times$ PI $\times$ positive/negative probe type) ANOVA was conducted on latencies, calculated as for Experiment 1. The set size factor was partitioned into three planned comparisons; set size 4 versus each of the remaining set sizes, set size 6 versus set sizes 8 and 10 , and set size 8 versus 10 . There was an effect of set size 4 versus set sizes 6,8 , and $10[F(1,189)=$ $104.25, p<.001]$; an effect of set size 6 versus 8 and $10[F(1,189)=34.56, p<.001] ;$ and of set size 8 with $10[F(1,189)=7.66, p<.01 ; M S e=18,775$ in all cases]. The set size main effect is equivalent to a scan rate of $23 \mathrm{msec}$ per item.

The main effect of PI yielded $F(1,63)=20.70, M S e$ $=19,655, p<.001$. This factor interacted with the comparison of set size 4 with the remaining set sizes $[F(1,189)$ $=4.60, M S \mathrm{e}=14,619, p<.05]$, but not with the other set size comparisons. The full set size $\times$ PI interaction is plotted in Figure 2. The presence of a PI effect at set sizes 6,8 , and 10 suggests that retrieval processes become operative at these set sizes. This implies that PM can hold only 4 items. The maximum set size used by

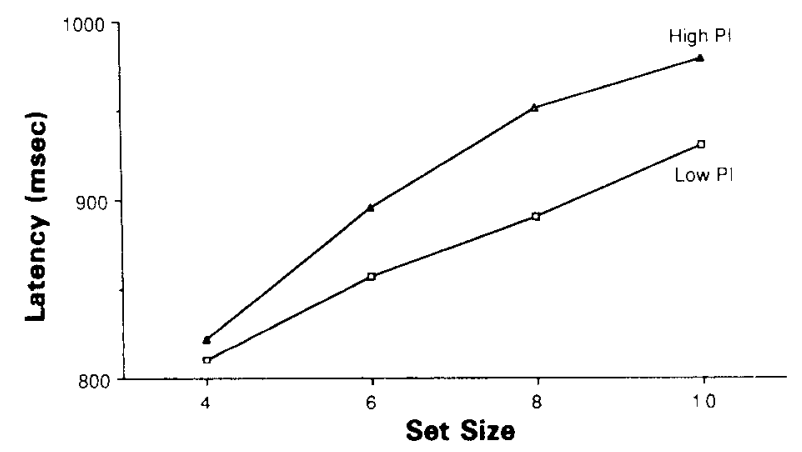

Figure 2. Interaction of set size and proactive interference (PI) on latency in Experiment 2.
Table 1

Set Size $\times$ Probe Type Interactions on Latencies and Proportion Correct in Experiment 2

\begin{tabular}{lllll}
\hline & \multicolumn{4}{c}{ Set Size } \\
\cline { 2 - 5 } Probe Type & 4 & 6 & 8 & 10 \\
\hline \multirow{4}{*}{ Latency (msec) } & \\
Negative & 799 & 877 & 929 & 960 \\
Positive & 834 & 875 & 913 & 949 \\
& & & & \\
Negative & .978 & .956 & .926 & .920 \\
Positive & .936 & .850 & .795 & .746 \\
\hline
\end{tabular}

Wickens et al.(1981; Wickens et al., 1985) was 4 items. Note that the sample was smaller than in Experiment 1, suggesting that the changed procedure produced marginally greater power.

As in Experiment 1, the hypothesis that the larger set sizes might have suffered greater PI because of longer delay was checked by examining serial position, but no effects were found. This hypothesis would also imply an interaction between PI and set size (6 vs. 10). This effect also was found nonsignificant $(F<1)$. This alternative interpretation of the set size $\times$ PI interaction therefore appears to be eliminated.

The failure to find this interaction might appear surprising, if we reason that the larger the set, the more items would have to be retrieved from SM. It is possible, however, that when sets were too large to be retained in PM, participants always retrieved the entire set, in preference to using subsets from two memory systems. This would mean that retrieval, and consequently PI, effects would be the same for all set sizes above 4 .

The positive/negative probe type main effect was not significant, but this factor interacted with set size 4 versus set sizes 6,8 , and $10[F(1,189)=6.63, M S e=14,161$, $p<.05]$. The full set size $\times$ probe type interaction is presented in Table 1 . The general form of this interaction indicates a steeper slope for negative than for positive probes, such that negative probes at set size 4 are responded to more quickly than positive probes, whereas the reverse is true at set sizes 8 and 10 .

This effect could be the result of two influences in the present study. First, as noted in Experiment 1, there may have been a bias toward negative responses that would have offset any general tendency to longer latencies with negative probes. Second, according to our hypothesis, retrieval will be required at larger set sizes, and negative probes are poorer retrieval cues, as Wickens et al. (1981) noted. Therefore, latencies should be relatively longer for negative probes at larger set sizes. The interaction in Table 1 is consistent with this, although the possibility of a trade-off with accuracy, to be discussed below, cannot be ruled out (see Table 1). Wickens et al. (1981) found longer latencies for negative probes in the SM condition and, as already noted, their set sizes were of 4 or fewer items. In our data, negative probes have shorter laten- 
cies than positive probes at set size 4 , but longer latencies at larger set sizes. The first effect is attributable to a generalized negative bias that is evident in all our data. The remainder of the effect is consistent with Wickens et al.'s findings, given that the larger set sizes require retrieval from SM, resulting in longer latencies for negative probes, just as Wickens et al. found longer latencies for negative probes in the SM condition.

Positive/negative probe type also interacted with PI $[F(1,63)=23.90, M S e=9,445, p<.001]$. Mean latencies for the low-PI condition were $856 \mathrm{msec}$ for negative trials and $888 \mathrm{msec}$ for positive trials, and mean latencies for the high-PI condition were $926 \mathrm{msec}$ for negative trials and $898 \mathrm{msec}$ for positive trials. This interaction should be interpreted with caution because it did not occur in Experiment 1. Shorter latencies for the negative probes in the low-PI condition are consistent with the negative response bias observed previously. However, in the high-PI condition, the negative probe produced much longer latencies than would be expected. This could occur if negative responses invariably require an attempt at retrieval of the set, whereas positive responses may sometimes occur because the probe matches an item in PM. This would cause PI to have a greater effect on negative probes.

An additional analysis of latencies on the high-PI trials was conducted with the factors of set size, positive/negative probe type, and new/old probe type in order to examine the effect of the last factor. The new findings of interest were a main effect of new/old probe type $[F(1,63)$ $=11.28, M S e=60,472, p<.001]$, and this factor interacted with positive/negative probe type $[F(1,63)=$ 20.34, MSe $=64,483, p<.001]$. Mean latencies for negative trials were $888 \mathrm{msec}$ for new probes and $1,011 \mathrm{msec}$ for old probes, and mean latencies for positive trials were $956 \mathrm{msec}$ for new probes and $936 \mathrm{msec}$ for old probes. This interaction reflects a tendency for old probes to yield longer latencies on negative trials and shorter latencies on positive trials. Old probes would tend to be retrieved from previous sets, producing a positive recognition response that would be appropriate on positive trials, but tending to conflict with the correct response on negative trials.

Accuracy. A $4 \times 2 \times 2$ (set size $\times$ PI $\times$ positive/negative probe type) ANOVA was performed on proportion correct. This yielded a main effect of set size 4 versus set sizes 6,8 , and $10[F(1,189)=182.40, p<.001]$; an effect of set size 6 versus set sizes 8 and $10[F=60.49$, $p<.001]$; and an effect of set size 8 versus set size 10 $[F=10.84, p<.01 ; M S e=.0088$ in all cases $]$. There was also a main effect of positive/negative probe type $[F(1,63)=102.52, M S e=.032, p<.001]$. However, positive/negative probe type interacted with all three set size comparisons: with set size 4 versus set sizes 6,8 , and $10[F(1,189)=53.41, p<.001]$; with set size 6 versus set sizes 8 and $10[F(1,189)=11.93, p<.001]$; and with set size 8 versus set size $10[F(1,189)=7.10, p<$ .001$, MSes $=.008]$. These data are shown in Table 1 . This interaction is of the same form as one obtained in
Experiment 1 . There is an overall tendency to give more correct responses to negative probes, possibly reflecting a bias toward negative responses (as noted previously), and this effect is more pronounced with large set sizes, possibly reflecting greater uncertainty. As noted previously, it could also, to some extent, reflect a trade-off with latency.

There was a main effect of PI $[F(1,63)=19.07, M S e$ $=.008, p<.001]$. The high-PI mean was .88 , and the low-PI mean was .90 . There was no significant interaction between set size and PI, but the means will be reported to show that the set size $\times$ PI interaction on latencies was not due to a trade-off with accuracy. In the lowPI condition, the mean proportions correct for set sizes $4,6,8$, and 10 were $.97, .91, .87$, and .85 , respectively; for the high-PI condition, the corresponding means were $.95, .89, .85$, and .82 .

An additional analysis of proportions correct on the high-PI trials was conducted with the factors set size, positive/negative probe type, and new/old probe type. The main new findings were a main effect of positive/negative probe type $[F(1,63)=54.99, M S e=.049, p<$ $.001]$, and of new/old probe type $[F(1,63)=6.33, M S \mathrm{Se}$ $=.025, p<.05]$. There was a significant interaction between these two factors $[F(1,63)=31.08, M S e=.020$, $p<.001]$. Mean proportions correct for negative trials were .96 for new probes and .89 for old probes, and mean proportions correct for positive trials were .81 for new probes and .84 for old probes. This interaction presumably reflects the same processes as the corresponding one with latencies. Old probes would tend to be retrieved from previous sets, leading to more appropriate responses on positive than on negative trials. This would modify the tendency for negative probes to produce more correct responses. Serial position effects and the interaction of set size 6 versus set size 10 with PI were checked as for latencies, but no effects were found.

Word-span scores were subjected to a tercile split, and the latency and accuracy analyses were repeated with this factor included. If PM capacity were higher for participants with higher spans, then PI should first occur at larger set sizes, leading to a span $\times$ set size $\times$ PI interaction. No such effect was observed. Correlations between span and size of the PI effect were checked at each set size and overall, but were not significant. However, lack of sensitivity and the small range of abilities mean that no firm conclusions should be drawn on this issue at this point. The mean span $(4.63, S D=.65)$ falls on the dividing line between set sizes that do not produce PI and set sizes that do. It is possible that span corresponds to the limit of capacity of PM, but the present data can only be suggestive on this point.

\section{EXPERIMENT 3}

The purpose of Experiment 3 was to see whether PM capacity is less for children. Set sizes 2 and 4 were used, with a design and procedure similar to that of Experiment 2 . 


\section{Method}

Participants. Forty-eight children from a middle-class primary school in Brisbane, Australia, participated. Their mean age was 8 years, 10 months (range $=8,1$ to 9,7 ).

Apparatus. The apparatus were the same as in Experiment 1.

Materials. The materials were as for Experiment 2, except there were only 16 categories plus 1 practice category, each of 11 words. The words that were most difficult to read, or were least common, or were of more than one syllable were eliminated.

Design and Procedure. The design and procedure were as for Experiment 2, except for the following changes: (1) set sizes of 2 and 4 items were used; (2) test blocks ( 2 through 6 ) contained only 24 test trials; (3) breaks were given at the end of any of the 6 blocks, if required; (4) to ensure that six words always preceded the high-PI trial, the three trials within a category used one of the following sequences of set sizes: $2-4-2,2-4-4,4-2-2$, or 4-2-4; and (5) the span test began with set size 3 .

\section{Results and Discussion}

Latency. A $2 \times 2 \times 2$ (set size $\times$ PI $\times$ positive/negative probe type) ANOVA was conducted on latencies, calculated as for Experiment 1. There were significant main effects of set size $[F(1,47)=72.76, M S e=23,505, p<$ $.001]$ and of PI $[F(1,47)=4.27, M S e=64,477, p<$ $.05]$, and a significant interaction of these two factors $[F(1,47)=5.03, M S e=64,344, p<.05]$. The means are shown in Figure 3. An additional analysis of latencies on the high-PI trials was conducted with the factors set size, positive/negative probe types, and new/old probe types as in Experiment 2 . The new finding was a main effect of new/old probes $[F(1,47)=$ $11.54, \mathrm{MSe}=119,241, p<.001]$. The mean latency on new probes was $1,332 \mathrm{msec}$ and on old probes was $1,451 \mathrm{msec}$.

Accuracy. A $2 \times 2 \times 2$ (set size $\times$ PI $\times$ positive/negative probe type) ANOVA was performed on proportion correct. This yielded a main effect of set size $[F(1,47)$ $=59.2, M S e=.0427, p<.001]$, of PI $[F(1,47)=5.19$, $M S e=.0582, p<.05]$, and of negative/positive probes $[F(1,47)=5.45, M S e=.1032, p<.05]$. There were no significant interactions, but we will report the four means for the set size $\times$ PI interaction to show that the corresponding interaction on latencies is not due to a tradeoff. The mean proportions correct for set size 2 were .97 for low PI and .96 for high PI, and mean proportions correct for set size 4 were .93 for low PI and .89 for high PI. The positive mean was .93 , and the negative mean was .95 .

Proportions correct were analyzed on high-PI trials, as for latencies. The new findings were a main effect of new/old probes $[F(1,47)=4.93, M S e=.0601, p<$ $.05]$, an interaction of new/old probes with negative/positive probes $[F(1,47)=8.70, M S e=.0902, p<.01]$, and an interaction of both these factors with set size $[F(1,47)=5.55, M S e=.0772, p<.05]$. The means are shown in Figure 4. The interaction of new/old probe type with negative/positive probe type has the same form as the corresponding interaction in Experiment 2, where it was interpreted as reflecting easier retrieval of old probes, leading to more appropriate responses on positive than on negative trials. The interaction of these factors with set size shows that the effect is greater with set size 4, which is consistent with the hypothesis that retrieval is more important with set size 4.

Word spans were examined as for Experiment 2, and were not found to be associated with PI effects. The mean word span was $3.53(S D=.48)$. Thus the mean again falls between set sizes that produce PI and those that do not.

\section{GENERAL DISCUSSION}

The conclusion of Wickens et al. (1981; Wickens et al., 1985 ) that proactive interference occurs only when information has been removed from consciousness by intervening activity must be modified in the light of these results. All of our experiments employed the PM paradigm used by Wickens and colleagues in that the

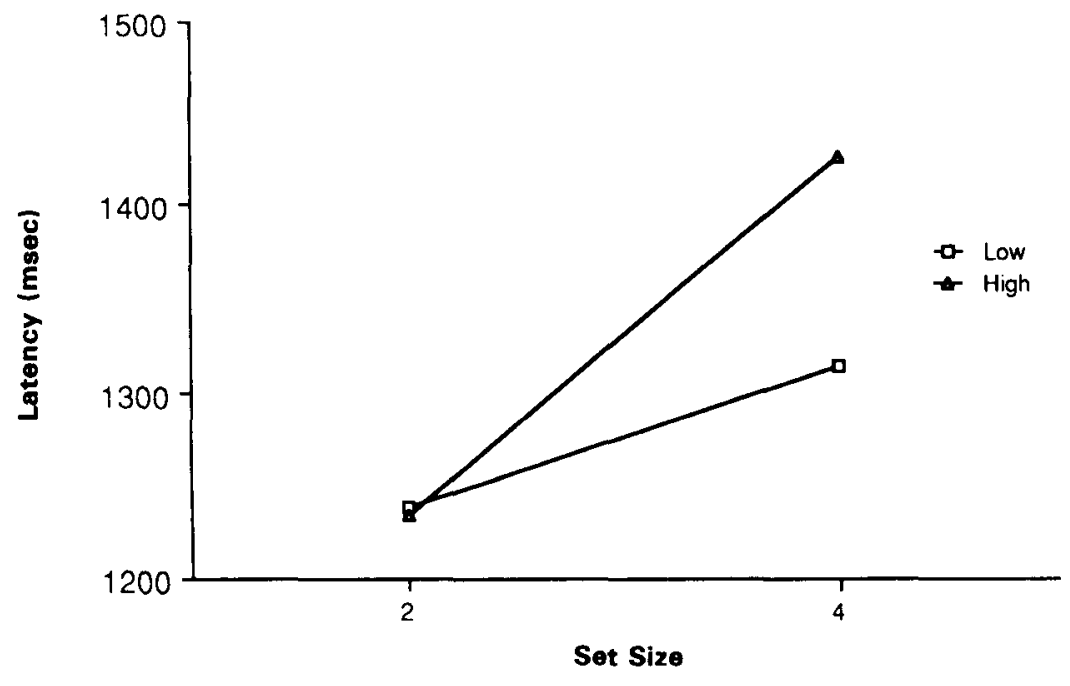

Figure 3. Interaction of set size and proactive interference for latency in Experiment 3. 


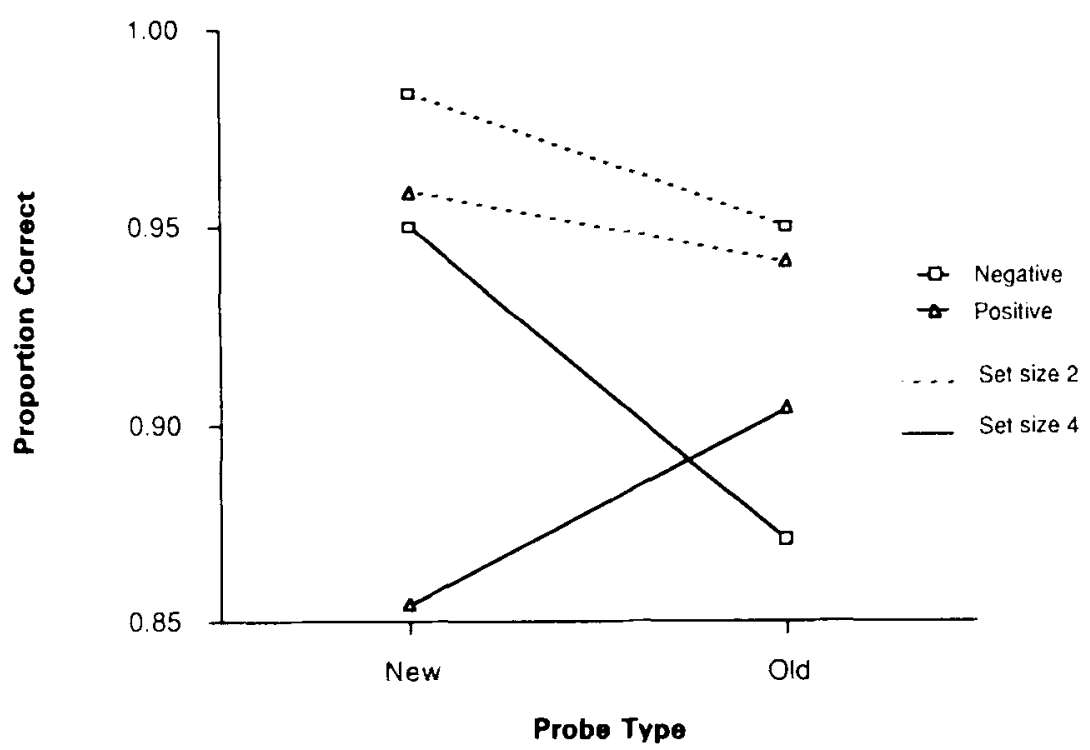

Figure 4. Interaction of new/old probe type with positive/negative probe type with set size on proportion correct in Experiment 3.

probe followed memory set presentation without intervening activity. However, whereas Wickens and colleagues found no PI in this condition, PI did occur in our data with set sizes above 4 items for adults, and with set size 4 for children. The findings of Wickens and colleagues, therefore, are restricted to small sets. There was a set size $x$ PI interaction on both errors and latencies in Experiment 1 , and on latencies in Experiments 2 and 3. The lack of an effect on accuracy in Experiments 2 and 3 may be attributed to the fact that the instructions and training for those experiments emphasized accuracy, and participants who failed to meet the required accuracy criterion were eliminated.

Flexser (1978), using prelearned supraspan lists (16 or 32 items), found no difference in the slope of the scanning function between PM and SM conditions, defined in terms of rehearsal prior to the probe. However, there was an intercept difference, which Wickens et al. (1981) interpreted as indicating that the items were in an active state in the PM condition. Our data suggest that only a small proportion of these items would have been in active memory, but this may have been sufficient to give an advantage in the PM condition. However, the number of procedural differences between Flexser's and our own research makes further comparison difficult.

The use of absence of proactive interference as a criterion for information's being in PM provides a new technique for investigating the capacity of active memory. Previous techniques have entailed the memory span task, working memory studies, and the recency portion of the free recall curve. The size of memory span is related to a number of cognitive performances (Dempster, 1981; Humphreys, Lynch, Revelle, \& Hall, 1983), but much of the variance in span is due to rehearsal rate (Baddeley, Thomson, \& Buchanan, 1975; Schweickert \& Boruff, 1986; Standing, Bond, Smith, \& Isely, 1980). Span pos- sibly reflects the operation of a relatively specialized system, a phonemic buffer or articulatory loop, and may not necessarily indicate the number of items that can be held in consciousness. This hypothesis is supported by working memory research, in which it has been found that reasoning interferes only minimally with retention of information in short-term memory (Baddeley, 1981; Klapp, Marshburn, \& Lester, 1983). In our data, no relationship was found between span and PM capacity, but this would not rule out a relationship being obtained when the technique becomes more refined, or if a larger range of spans were used. The recency portion of the serial position curve is also unlikely to be related to the number of items that can be held in consciousness, because it is unrelated to cognitive processing (Baddeley \& Hitch, 1974).

The findings do not have to be interpreted within a strict two-store model. It is possible that items that we have identified as being in PM are actually distinguished by high trace strength, in the manner of the model by Baddeley and Ecob (1973). If the effect of increasing the number of items in the set is to decrease the trace strength of the items, they will become less distinguishable. Alternatively, the data could be interpreted in terms of models of distributed information storage, such as those by Murdock (1982), Rumelhart and McClelland (1986), or Pike, Dalgleish, and Wright (1977). In this category of models, capacity limitations arise because, as the number of stimuli represented increases, while the number of representational units are held constant, the items become less distinguishable. In the context of these models, an active memory capacity limitation would occur because any one pattern of primary activation can represent only a limited number of items with clarity. The present research provides no basis for distinguishing different theories of information storage, but our data give grounds for the speculation that such models should incorporate 
a capacity limitation equivalent to four or five items being activated at one time.

A question that is of considerable significance in cognitive development concerns whether capacity to process information changes with age (Case, 1985; Halford, Maybery, \& Bain, 1986; Kail, 1986). Use of proactive interference to test the capacity of PM provides a new approach for investigating this issue. Experiment 1 found no evidence of an age effect, but the younger sample in Experiment 3 produced evidence that primary memory capacity is less for younger participants.

It is noticeable that the mean span falls between set sizes that produce PI and those that do not in both Experiments 2 and 3. Only further research will establish whether this is coincidence, but Schweickert and Boruff (1986) offered a speculation that would explain why this might be the case, even though span and cognitive processing utilize separate systems, as suggested above. Their suggestion is that the capacity of the central processor might be matched to the capacity of the phonemic buffer used in span, because there would be no point in having a string of items in the buffer that could not be scanned. Thus span size might correspond to the number of items that can be in an active state in the processor, even though span occupies a different system.

The present research indicates that with a varied set procedure, no more than 5 words can be held in active memory by adults and teenage children, or 3 words by 8- to 9-year-olds. This is indicated by the finding that proactive interference, found to be associated with secondary but not primary memory by Wickens et al. (1981; Wickens et al., 1985), occurs at set size 6 and above for adults, and at set size 4 for children, even where there is no intervening activity that might have removed the items from consciousness. This finding is consistent with an overload of PM. The set size $\times$ PI paradigm might provide a means of investigating the capacity of $P M$, and whether it changes with age or with psychopathology. Even if the test lacks the economy of other measures, such as span, it could be used to establish whether those other measures are related to the number of items that can be held in an active state.

\section{REFERENCES}

BADDELEY, A. (1981). The concept of working memory: A view of its current state and probable future development. Cognition, 10, 17-23.

BADDELEY, A. D., ECOB, J. R. (1973). Reaction time and short-term memory: Implications of repetition effects for the high-speed exhaustive scan hypothesis. Quarterly Journal of Experimental Psychology, 25, 229-240.

BADDELEY, A. D., \& Hitch, G. (1974). Working memory. In G. H. Bower (Ed.), The psychology of learning and motivation: Advances in research and theory (pp. 47-89). New York: Academic Press.
Baddeley, A. D., Thomson, N., Buchanan, M. (1975). Word length and the structure of short-term memory. Journal of Verbal Learning \& Verbal Behavior, 14, 575-589.

Battig, W. F., \& Montague, W. E. (1969). Category norms for verbal items in 56 categories: A replication and extension of the Connecticut category norms. Journal of Experimental Psychology Monographs, 80(3, Pt. 2).

CASE, R. (1985). Intellectual development: Birth to adulthood. New York: Academic Press.

Dempster, F. N. (1981). Memory span: Sources of individual and developmental differences. Psychological Bulletin, 89, 63-100.

FLEXSER, A. J. (1978). Long-term recognition latencies under rehearsalcontrolled conditions: Do list-length effects depend on active memory? Journal of Experimental Psychology, 4, 47-54.

Gardiner, J. M., Craik, F. I. M., BirTWhistle, J. (1972). Retrieval cues and release from proactive inhibition. Joumal of Verbal Learning \& Verbal Behavior, 11, 778-783.

Halford, G. S., Maybery, M. T., \& Bain, J. D. (1986). Capacity limitations in children's reasoning: A dual task approach. Child Development, 57, 616-627.

Humphreys, M. S., Lynch, M. J., Revelle, W., \& Hall, J. W. (1983). Individual differences in short-term memory. In R. F. Dillon \& R. R. Schmeck, Individual differences in cognition (pp. 3564). New York: Academic Press.

James, W. (1890). Principles of psychology. New York: Holt, Rinehart and Winston.

KaIL, R. (1986). Sources of age differences in speed of processing. Child Development, 57, 969-987.

Klapp, S. T., Marshburn, E. A., \& Lester, P. T. (1983). Short-term memory does not involve the "working memory" of information processing: The demise of a common assumption. Joumal of Experimental Psychology: General, 112, 240-264.

MURDOCK, B. B., JR. (1982). A theory for the storage and retrieval of item and associative information. Psychological Review, 89, 609-626.

Pike, R., Dalgleish, L., \& Wright, J. (1977). A multiple-observations model for response latency and the latencies of correct and incorrect responses in recognition memory. Memory \& Cognition, 5, 580-589.

Rumelhart, D. E., \& McClelland, J. L. (Eds.). (1986). Parallel distributed processing: Explorations in the microstructure of cognition (Vol. 1). Boston, MA: MIT Press.

SCHWEICKeRT, R., BoRUFF, B. (1986). Short-term memory capacity: Magic number or magic spell? Journal of Experimental Psychology: Leaming, Memory, \& Cognition, 12, 419-425.

Standing, L., Bond, B., Smith, P., \& Isley, C. (1980). Is the immediate memory span determined by subvocalization rate? British Journal of Psychology, 71, 525-539.

STERNBERG, S. (1975). Memory scanning: New findings and current controversies. Quarterly Journal of Experimental Psychology, 27, $1-32$.

WAtKINS, O. G., * WatkINS, M. J. (1975). Buildup of proactive interference as a cue-overload effect. Journal of Experimental Psychology: Human Learning \& Memory, 104, 442-452.

WiCKenS, D. D., Moody, M. J., Dow, R. (1981). The nature and timing of the retrieval process and of interference effects. Journal of Experimental Psychology: General, 110, 1-20.

WICKENS, D. D., Moody, M. J., \& VIDULICH, M. (1985). Retrieval time as a function of memory set size, type of probes, and interference in recognition memory. Journal of Experimental Psychology, 11, 154-164.

(Manuscript received April 6, 1987; revision accepted for publication January 19, 1988.) 